\title{
Beacon-driven Leader Based Protocol over a GE Channel for MAC Layer Multicast Error Control
}

\author{
Zhao LI ${ }^{1}$, Thorsten HERFET ${ }^{2}$ \\ ${ }^{1}$ Student Member IEEE, USTC CS China \& Saarland University, Germany \\ ${ }^{2}$ Senior Member IEEE, Saarland University, Germany \\ E-mail:\{li,herfet\}@nt.uni-saarland.de
}

\begin{abstract}
In wireless networks current standard MAC layer protocols don't provide any error correction scheme for broadcast/multicast. In this paper, we enhance a Leader Based Protocol (LBP) and propose a Beacon-driven Leader Based Protocol (BLBP) for the MAC layer multicast error control. To guarantee a very low Packet Loss Ratio (PLR) under strict delay constraints for video multicast over a Gilbert-Elliott (GE) channel, we analyze BLBP and compare it with LBP and different application layer multicast error control schemes via simulation experiments. Both the theoretical analysis and simulation results show that BLBP can correct nearly all the errors for all receivers in the MAC layer and is more efficient than LBP. BLBP is also more efficient than the application layer Automatic Repeat request (ARQ) scheme and the total multicast delay is much shorter. BLBP is very good for real-time multicast applications with strict delay constraints.
\end{abstract}

Keywords: BLBP, Multicast Error Control, Gilbert-elliott Channel

\section{Introduction}

With the rapid development of wireless networking technologies, it is becoming possible to supply wireless terminal users not only with data connections, but also with real-time communication services. The emerging real-time multicast applications in wireless networks include the local distribution of High Definition TV (HDTV) and Digital Video Broadcasting (DVB) [1], video-on demand, video conferencing, gaming, local VoIP, IPTV, Internet-Radio distribution, P2P broadcasting, etc. Most of these applications require a very low Packet Loss Ratio (PLR) under strict delay constraints.

The characteristics of wireless network can be summarized as a bandwidth variation and terminal heterogeneity plus a high degree of packet losses. It is known that the Gilbert-Elliot (GE) channel $[2,4]$ with a 2-state Markov model is a good approximation for the packet loss model in wireless channels. However, the current standard MAC layer protocols don't provide any error correction scheme for broadcast/multicast. Hence the multicast error is controlled in the application layer. The existing application layer multicast error control schemes include automatic repeat request (ARQ), forward error correction (FEC) and hybrid error correction (HEC) [5-10]. Unfortunately the total multicast delays of the application layer schemes are always high and sometimes do not satisfy the strict application delay constraints, or these schemes are not efficient when the delay constraints are short.

Compared with application layer schemes, MAC layer multicast error control schemes recover the multicast loss locally and lead to much shorter delays. Currently, very few reliable MAC layer multicast schemes, such as Leader Based Protocol (LBP) [11], have been proposed for IEEE 802.11 based wireless networks. LBP elects one of the multicast group receivers as the leader. On erroneous reception of a data frame, the leader does not send an acknowledgement (ACK), prompting a retransmission. On erroneous reception of the data frame at the non-leader receivers, LBP allows negative acknowledgements (NACKs) from these receivers to collide with the ACK from the leader, thus destroying the ACK and prompting the sender to retransmit the data frame. We refer to this ACK/NACK jam as JACK.

However, LBP suffers from two main problems: First, when the entire data frame is lost, the non-leader 
receivers can not reply NACKs because they don't know when or how to send them, as the destination is unknown especially when Request-To-Send (RTS) and Clear-To-Send (CTS) is not used for small data frames. As a result, LBP is not reliable for the non-leader receivers. Second, LBP has poor performance when the channel error rates are high. The non-leader receivers send NACKs whenever the received frame is in error, regardless of whether this erroneous frame has been received correctly before or not. This is because the receivers in LBP can not access the data frame sequence number before the frame is received, as there is no such field in the structure of RTS/CTS frames for multicast. So the sender has to retransmit until all receivers receive the data frame correctly at the same time. There are a lot of unnecessary transmissions; hence LBP is not efficient particularly for lossy channels.

In this paper, we enhance LBP and propose a Beacon-driven Leader Based Protocol (BLBP). The sender sends a beacon frame before the data frame to lead the non-leader receivers to set timers and to announce the sequence number of the following data frame. BLBP solves the problems of LBP well. Each of the non-leader receivers can send feedback when the timer times out. Both the leader receiver and non-leader receivers can send ACK and NACK respectively based on sequence check, hence avoids the unnecessary transmissions in LBP. To guarantee a very low PLR under strict delay constraints for video multicast over a GE channel, we analyze BLBP and compare it with LBP and two application layer multicast error control schemes via simulation experiments. One is an ARQ based scheme from [8], called HEC-PR, which combines a NACK based ARQ scheme with a packet repetition (PR) technique. The other one is a nearly optimal application layer multicast error control scheme called Hybrid ARQ (HARQ) Type I [9,10], which combines FEC and NACK based ARQ scheme together.

The remainder of this paper is organized as follows. Section 2 presents the related work. In section 3, we examine why it is necessary to correct multicast errors in the MAC layer. We describe BLBP in section 4 and analyze its performance over a GE channel in section 5 . And in section 6, we evaluate the performance of BLBP and compare it with LBP, HEC-PR and HARQ Type I via simulation experiments. We conclude in section 7 .

\section{Related Work}

For the application layer multicast error control, many authors [5,8] studied the ARQ based schemes and concluded that when combined with feedback suppression and other accessorial techniques, ARQ is effective to repair multicast packet losses for small groups with low error rates. However, the application layer ARQ always take a long time and they are not efficient at high error rates and with large numbers of receivers due to feedback implosion and the limitation to scale.

Another technique commonly used to handle losses for multicast in the application layer is FEC, whereby redundant information in the data stream enables the receiver to correct losses without contacting the sender. Rizzo [6] studied the feasibility of software encoding/decoding for packet-level FEC. A $(n, k)$ block erasure code converts $k$ source packets into a group of $n$ coded packets, such that any $k$ of the encoded packets can be used to reconstruct the $k$ source packets. Usually, the first $k$ packets in each group are identical to the original $k$ data packets; the remaining $n-k$ packets are referred to as parity packets. The advantage of using block erasure codes for wireless multicasting is that a single parity packet can be used to correct independent single-packet losses among different receivers.

The integrated FEC/ARQ schemes or any other kinds of combination of more than one error control schemes are referred to as HEC schemes in this paper. Previous works [7-10] indicate that HEC schemes are much more efficient for recovering data packets than the schemes with either FEC or ARQ alone. We consider the HECPR scheme from [8], which combines a NACK based ARQ scheme and a packet repetition technique. The number of feedback/retransmission rounds and the number of packet repetitions in each round are adapted to the network condition. HEC-PR is actually an ARQ based scheme without FEC coding. We also consider HARQ Type I from $[9,10]$, in which the sender sends a certain amount of parity packets using FEC following the original $k$ data transmissions. If the loss rate obtained after reconstruction at the receiver is still too high, ARQ is used to retransmit more parity packets. Tan [10] developed formulas to optimize the performance of HARQ Type I while guaranteeing the required PLR under strict delay constraints. HARQ Type I is a nearly optimal application layer multicast error control scheme.

However, these application layer multicast schemes always take long multicast delays or they are not efficient when the delay constraints are short. So we study MAC layer multicast error control schemes to support real-time multicast with strict delay constraints. For the reliable MAC layer multicast, besides LBP [11], Tourrihes [12] proposed a robust broadcast using a collision detector to inform the sender whether the broadcast packet is successful or not. However, this scheme can not guarantee the reliability of multicast transmissions because the feedbacks are only from the detector instead of all receivers themselves. Gupta et al. [13] proposed a tone-based solution for multicast in both infrastructure and ad-hoc 802.11 networks. They use dual busy tones to simulate NACKs or Negative CTS (NCTS). Although this scheme is good to detect and correct the multicast errors, it requires an additional 
channel for the tone, which is not always feasible in practice.

Our BLBP enhances LBP with a beacon frame to lead the non-leader receivers to set timers and to announce the sequence number of the following data frame. BLBP avoids the problems of LBP and is more efficient.

\section{Motivation}

The emerging real-time multicast applications in wireless networks (such as wireless HDTV, DVB, game, video conference) require strict delay constraints. Error recovery based on application layer ARQ is suboptimal because the end-to-end application layer feedback and retransmission take a too long time due to application layer protocol waiting, MAC layer queuing, hardware handling, etc. Moreover, the application layer ARQ based schemes are not efficient when the error rates are high and the numbers of receivers are large due to feedback implosion and the limitation to scale. The FEC coding based application layer schemes can satisfy the strict delay constraints but they are not efficient when the delay constraints are very strict particularly for small multicast groups. And the FEC based ones are not adaptive to the heterogeneity of receivers because the code has to be set based on the receiver with the worst channel condition.

Compared with application layer schemes, MAC layer multicast error control schemes take a much shorter time due to the faster feedback and retransmission. Due to the JACK scheme, BLBP and LBP even achieve complete feedback suppression. So the MAC layer multicast error control schemes are very good for real-time multicast applications. For non-realtime multicast applications such as file dissemination and shared whiteboards, reliable MAC layer multicast saves time as well as both network and end-system resources.

Moreover, for multi-hop multicast with wired network and wireless LAN as the last hop, the need for additional transmissions due to errors in the wireless LANs puts unnecessary processing burden on the original remote sender. These additional transmissions go over the entire wired multicast tree and also the wireless links, taking a long time, wasting bandwidth and also leading to processing of unwanted redundant retransmissions at those receivers which might have already received the packet. The similar thing also happens in multi-hop wireless networks such as wireless mesh networks and wireless ad hoc networks. If the access points (AP) (or base stations) were to take the responsibility of supplying retransmissions rather than the original sender, then the load of supplying retransmission gets distributed across access points and takes a shorter time. The total error correction cost will be much shorter and it is easier to guarantee the final PLR under strict delay constraints in the application layer.

\section{Main Scheme of BLBP}

The MAC layer reliable multicast BLBP requires a slight modification to the IEEE 802.11 MAC layer protocols. As mentioned earlier, 802.11 DCF (Distributed Coordination Function) unicast - assumed RTS/CTS is switched on to solve the hidden terminal problem - is more reliable than broadcast/multicast, because unicast uses RTS/CTS signaling and ACK/retransmission scheme in the MAC layer and broadcast/multicast does not.

BLBP enhances LBP with a MAC control frame called beacon shown in Figure 1. Besides the same fields in RTS/CTS frames, such as frame control header, transmission duration, receiver address (RA), transmitter address (TA) and frame check sequence (FCS), the beacon frame also includes the sequence number of the following data frame. The use of the beacon frame is to lead the non-leader receivers to set timers and to announce the sequence number of the following data frame.

\begin{tabular}{|c|c|c|c|c|c|}
\multicolumn{2}{c}{2} & \multicolumn{1}{c}{6} & 6 & 2 & 4 \\
\hline $\begin{array}{c}\text { Frame } \\
\text { Control }\end{array}$ & Duration & RA & TA & $\begin{array}{c}\text { Sequence } \\
\text { Control }\end{array}$ & FCS \\
\hline
\end{tabular}

Figure 1. The format of the beacon frame

The main scheme of BLBP is shown in Figure 2. A receiver is selected as the leader for the multicast group. The AP first sends a RTS frame to all receivers, and only the leader receiver transmits a CTS frame in reply to the AP. The AP is then assured that the channel is granted and starts the transmission of the beacon frame with the sequence number of the following data frame. On receipt of the beacon frame, each of the non-leader receivers sets a timer according to the beacon frame. The AP then transmits the data frame following the beacon frame. The leader receiver replies an ACK frame if the data is correct or it has already got the data based on sequence check, or does nothing otherwise. When the timer times out, each non-leader receiver replies a NACK if the data is error and it has not received it correctly yet based on sequence check, or does nothing otherwise. Then if the AP receives an ACK, this transmission is done. Otherwise, the AP repeats the whole procedure and retransmits again until the number of times is beyond the retransmission limit. For example, in the retransmission phase in Figure 2, although this time the data frame is lost, the leader receiver still replies an ACK because it knows this data frame has been received correctly already in the first transmission, thanks to the beacon frame.

For the determination of a leader, we use a scheme 
from LBP [11]: The first receiver that joins the multicast group acts as the leader. The group is cancelled if there is no leader. The other group members can rebuild/rejoin the multicast group if necessary when time out. Please note that it is possible to reduce the amount of control traffic flow for leader election purposes when a higher layer group management protocol like IGMP (Internet Group Management Protocol) is running above the link layer [11]. The leader does not affect the performance because BLBP supplies fair service for all receivers.

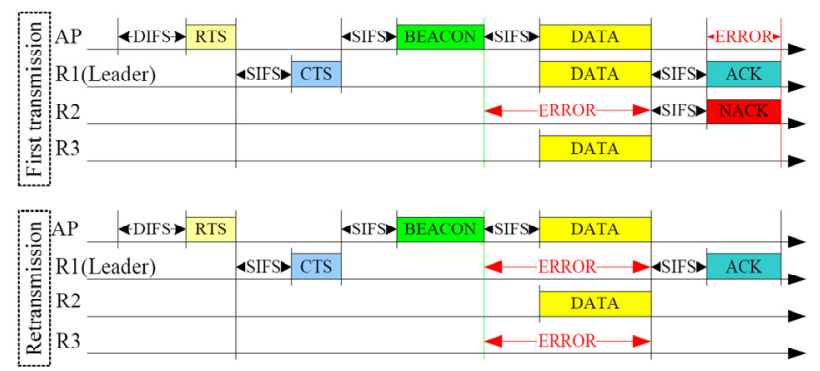

Figure 2. Main scheme of BLBP (Ri denotes receiver i)

BLBP solves the problems of LBP well. All the nonleader receivers can send feedbacks when the timers time out. Both the leader receiver and non-leader receivers send ACK and NACK respectively based on sequence check thanks to the beacon frame, hence it avoids the unnecessary transmissions in LBP. Clearly, BLBP can correct all the errors for all receivers due to the ACK/NACK feedback and retransmission in the MAC layer. BLBP is even more efficient than the application layer ARQ schemes because BLBP suppresses the multiple feedbacks into just a JACK. However, the loss of beacon frames will decrease the performance (reception rate) of the non-leader receivers. Fortunately, the beacon frames are much more reliable (nearly error free) than data frames because they are much smaller and are transmitted using the lowest data rate, like other control frames in 802.11 (RTS, CTS, ACK). Moreover, due to RTS/CTS signaling, the beacon frames also avoid collision loss.

Please also note that BLBP can run without RTS/CTS exchanges for small data frames just like 802.11 DCF unicast. Although our discussion is in the context of 802.11 DCF, BLBP is actually applicable to all ACK/retransmission based MAC protocols, such as 802.11 PCF (Point Coordination Function) etc.

\section{Performance Analysis}

In this section we first introduce the GE channel model and then analyze the theoretical performance of BLBP over the GE channel model with both temporal error correlation and spatial error correlation.

\subsection{GE Channel Model}

The GE channel model is a two-state Markov chain shown in Figure 3. In the Good state $(G)$ errors occur with (low) probability $P_{G}$ while in the Bad state (B) they occur with (high) probability $P_{B}$.

The errors occur in clusters or bursts with relatively long error-free intervals (gaps) between them. The state transition is summarized by its transition probability matrix in formula (1).

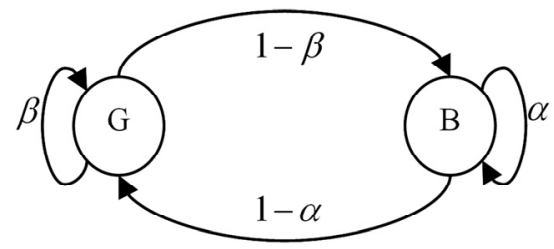

Figure 3. GE channel model

$$
P=\left[\begin{array}{cc}
\beta & 1-\beta \\
1-\alpha & \alpha
\end{array}\right]
$$

To reflect most reasonable choices for real scenarios, it is assumed that $P_{G}=0$ and $P_{B}=1$. This model is always referred to as the simplified GE model. Our analysis and simulation experiments in the following sections are based on the simplified GE model.

The occupancy times for state B and $G$ are both geometrically distributed with respective means $(1-\alpha)^{-1}$ and $(1-\beta)^{-1}$, which are also called as the expected error burst length and the expected error free length respectively. The steady state probabilities of being in states $\mathrm{G}$ and $\mathrm{B}$ are $\pi_{G}=(1-\alpha) /(2-\alpha-\beta)$ and $\pi_{B}=(1-\beta) /(2-\alpha-\beta)$ respectively. So the average packet loss rate produced by the GE channel model is

$$
p=P_{G} \pi_{G}+P_{B} \pi_{B}=\frac{P_{G}(1-\alpha)+P_{B}(1-\beta)}{(1-\alpha+1-\beta)}
$$

For the simplified GE channel model, the PLR will be

$$
p=\frac{(1-\beta)}{(1-\alpha+1-\beta)}
$$

Following [14], the variance of the error symbol (or packet) $X$ is $\sigma^{2}=E(X-\bar{p})^{2}=p(1-p)$. So we get the correlation of two consecutive error symbols $X_{I}$ and $X_{2}$ :

$$
\tau=\frac{E\left(\left(X_{1}-p\right)\left(X_{2}-p\right)\right)}{\sigma^{2}}=\alpha+\beta-1
$$

which is also referred to as the temporal error correlation.

Finally, the two parameters of the simplified model ( $\alpha$ and $\beta$ ) can be expressed in the terms of the more meaningful quantities $p$ and $\tau$ by solving formulas (3) and (4). These yields 


$$
\begin{aligned}
& \alpha=p+\tau(1-p) \\
& \beta=(1-p)+\tau p
\end{aligned}
$$

The transition probability matrix then becomes

$$
P=\left[\begin{array}{cc}
1-p(1-\tau) & p(1-\tau) \\
(1-p)(1-\tau) & 1-(1-p)(1-\tau)
\end{array}\right]
$$

And the $I$-step transition probability matrix is:

$$
P^{I}=\left[\begin{array}{cc}
1-p\left(1-\tau^{I}\right) & p\left(1-\tau^{I}\right) \\
(1-p)\left(1-\tau^{I}\right) & 1-(1-p)\left(1-\tau^{I}\right)
\end{array}\right]
$$

Now we compute $P[a, b]$, the probability of $a$ errors in a sequence of $b$ symbols following [14]. Let $P_{G}[a, b]$ be the probability of $a$ errors in $b$ transmissions with the channel ending in state G. Similarly, let $P_{B}[a, b]$ be the probability of $a$ errors in $b$ transmissions with the channel ending in state $\mathrm{B}$. Then

$$
P[a, b]=P_{G}[a, b]+P_{B}[a, b]
$$

For $b=1,2,3 \ldots$ and $a=0,1,2 \ldots b$, assuming the simplified GE channel, then

$$
\begin{aligned}
P_{G}[a, b] & =P_{G}[a, b-1] \beta+P_{B}[a, b-1](1-\alpha) \\
P_{B}[a, b] & =P_{B}[a-1, b-1] \alpha+P_{G}[a-1, b-1](1-\beta)
\end{aligned}
$$

The initial conditions for the recursion are

$$
\begin{gathered}
P_{G}[0,0]=(1-\alpha) /(2-\alpha-\beta) \\
P_{B}[0,0]=(1-\beta) /(2-\alpha-\beta)
\end{gathered}
$$

and $P_{G}[a, 0]=P_{B}[a, 0]=0$ for $a \neq 0$. Note that with these initial conditions, all numerical values computed will be steady state results.

\subsection{BLBP over the GE Channel Model with Temporal Error Correlation}

Now we analyze BLBP over the GE channel model. As in most referenced papers, it is assumed that the MAC layer control frames (RTS/CTS/Beacon/ACK) are error free from the error model. This also makes sense in practice because the control frames are very small and are sent in the lowest data rate, and hence they are more reliable than data frames. We first consider only the temporal error correlation. In other words, it is assumed that the error events at different receivers are independent. To be clear, we show the used symbols here.

- $P$ : The original packet error rate for all receivers;

- $R$ : The number of receivers;

- $m$ : The retransmission limit;
- $N$ : The total number of transmissions required to transmit a multicast packet correctly to all the $R$ receivers;

- $N_{r}$ : The number of transmissions required for receiver $r$ to receive a packet correctly;

- $P L R_{\text {target mac }}$ : The PLR target in the MAC layer;

- $D_{\text {target_mac }}$ : The Delay target in the MAC layer.

First we consider the final PLR for receiver $r$, shown in formula (14).

$$
P L R(r)=P[m+1, m+1]=p \alpha^{m}
$$

About the determination of the retransmission limit $m$, there are two constraints, the PLR constraints and the delay constraints which are shown as follows:

$$
\begin{aligned}
& p \alpha^{m} \leq P L R_{t_{\text {arget_mac }}} \\
& m^{*} T_{\text {BLBP }} \leq D_{\text {target_mac }_{\text {mac }}}
\end{aligned}
$$

where $T_{B L B P}=T_{C C}+T_{R T S}+T_{C T S}+T_{B E A C O N}+T_{D A T A}+T_{A C K}$ $+D I F S+4 S I F S$ is the time of one transmission in BLBP. $T_{C C}$ denotes the channel contention time which can be calculated theoretically following [15] or by measurements in practice. $T_{R T S}, T_{C T S}, T_{B E A C O N}, T_{D A T A}$, and $T_{A C K}$ are the transmission times of frames RTS, CTS, BEACON, DATA and ACK respectively. DIFS denotes the Distributed Inter Frame Space while SIFS is the Short Inter Frame Space. Note that the PLR target and the delay target may not be satisfied at the same time sometimes, especially when the delay constraint is too strict. We will explore this further in simulation experiments.

Now we consider the expected number of transmissions for one multicast data packet. The probabilities that $N_{r} \leq n, N_{r}=n$ and $N_{r}>n$ are shown in formulas (17), (18) and (19) respectively.

$$
\begin{aligned}
& P\left[N_{r} \leq n\right]=1-P[n, n] \\
= & \begin{cases}1-p \alpha^{n-1}, & n=1,2 \ldots m+1 \\
0, & n=0\end{cases} \\
& P\left[N_{r}=n\right]=P\left[N_{r} \leq n\right]-P\left[N_{r} \leq n-1\right] \\
= & \begin{cases}p \alpha^{n-2}-p \alpha^{n-1}, & n=2,3 \ldots m+1 \\
1-p, & n=1\end{cases} \\
& P\left[N_{r}>n\right]=P[n, n]= \begin{cases}p \alpha^{n-1}, & n=1,2 \ldots m \\
1, & n=0\end{cases}
\end{aligned}
$$

So we get the expected number of transmissions for one multicast data packet required for receiver $r$ :

$$
E\left(N_{r}\right)=\sum_{n=0}^{m} P\left[N_{r}>n\right]=1+\frac{p\left(1-\alpha^{m}\right)}{(1-\alpha)}
$$

Next the probabilities that $N \leq n, N=n$ and $N>n$ are shown in formulas (21), (22) and (23) respectively. 


$$
\begin{aligned}
& P[N \leq n]=\prod_{r=1}^{R} P\left[N_{r} \leq n\right] \\
= & \begin{cases}\left(1-p \alpha^{n-1}\right)^{R}, & n=1,2 \ldots m+1 \\
0, & n=0\end{cases} \\
& P[N=n]=P[N \leq n]-P[N \leq n-1] \\
= & \begin{cases}\left(1-p \alpha^{n-1}\right)^{R}-\left(1-p \alpha^{n-2}\right)^{R}, & n=2,3 \ldots m+1 \\
(1-p)^{R}, & n=1\end{cases} \\
& P[N>n]=1-P[N \leq n] \\
= & \begin{cases}1-\left(1-p \alpha^{n-1}\right)^{R}, & n=1,2 \ldots m \\
1, & n=0\end{cases}
\end{aligned}
$$

Finally we get the expected number of transmissions for one multicast data packet for all receivers, shown in formula (24).

$$
\begin{gathered}
E[N]=\sum_{n=0}^{m} P[N>n] \\
=1+\sum_{n=1}^{m}\left(1-\left(1-p \alpha^{n-1}\right)^{R}\right)
\end{gathered}
$$

Similarly, we get the expected number of transmissions for one multicast data packet for all receivers in LBP, shown in formula (25).

$$
\begin{aligned}
& E_{L B P}[N]=\sum_{n=0}^{m}\left(1-(1-P[1,1])^{R}\right)^{n} \\
= & \sum_{n=0}^{m}\left(1-(1-p)^{R}\right)^{n}
\end{aligned}
$$

And the redundant information (RI) of BLBP is:

$$
R I=E[N]-1
$$

Similar as in [16], among pure ARQ based multicast error control schemes, BLBP needs the minimum number of transmissions (shown in formula (24)) to let all receivers receive the packet correctly. There are no unnecessary transmissions due to the sequence check scheme and the complete feedback suppression based on the JACK scheme. We will explore this further in simulation experiments by comparing BLBP with LBP and an application layer pure ARQ scheme.

\subsection{BLBP over the GE Channel Model With Both Temporal and Spatial Error Correlation}

Now we consider BLBP over a GE channel model with both the temporal error correlation and the spatial error correlation. The error events at different receivers are in a certain correlation. We assume two kinds of error events, the error event at the sender which leads to the correlated packet loss among all receivers and the error events at different receivers which lead to independent packet losses. Some new symbols are shown as follows.

- $P_{m}$ : The error rate caused at receivers (called input error), independent of each other;

- $P_{\text {out }}:$ The error rate caused at the sender (called output error), which leads to correlated loss covering all receivers; $p=p_{\text {out }}+\left(1-p_{\text {out }}\right) p_{\text {in }}$;

- $P_{i n}=\left[\begin{array}{cc}\beta_{i n} & 1-\beta_{i n} \\ 1-\alpha_{i n} & \alpha_{i n}\end{array}\right]$ : The transition probability matrix of the input errors at all receivers;

- $P_{\text {out }}=\left[\begin{array}{cc}\beta_{\text {out }} & 1-\beta_{\text {out }} \\ 1-\alpha_{\text {out }} & \alpha_{\text {out }}\end{array}\right]$ : The transition probability matrix of the output error at the sender;

- $\lambda$ : The spatial error correlation among different receivers, $\lambda=p_{\text {out }} / p$.

Combining the output error and the input error, we can compute the total error model, which is a 4-states Markov chain, shown as follows.

$P=\left[\begin{array}{cccc}\beta_{\text {out }} \beta_{\text {in }} & \beta_{\text {out }}\left(1-\beta_{\text {in }}\right) & \left(1-\beta_{\text {out }}\right) \beta_{\text {in }} & \left(1-\beta_{\text {out }}\right)\left(1-\beta_{\text {in }}\right) \\ \beta_{\text {out }}\left(1-\alpha_{\text {in }}\right) & \beta_{\text {out }} \alpha_{\text {in }} & \left(1-\beta_{\text {out }}\right)\left(1-\alpha_{\text {in }}\right) & \left(1-\beta_{\text {out }}\right) \alpha_{\text {in }} \\ \left(1-\alpha_{\text {out }}\right) \beta_{\text {in }} & \left(1-\alpha_{\text {out }}\right)\left(1-\beta_{\text {in }}\right) & \alpha_{\text {out }} \beta_{\text {in }} & \alpha_{\text {out }}\left(1-\beta_{\text {in }}\right) \\ \left(1-\alpha_{\text {out }}\right)\left(1-\alpha_{\text {in }}\right) & \left(1-\alpha_{\text {out }}\right) \alpha_{\text {in }} & \alpha_{\text {out }}\left(1-\alpha_{\text {in }}\right) & \alpha_{\text {out }} \alpha_{\text {in }}\end{array}\right]$

For the convenience of analyzing, we use a GE channel model to approximate the total error model. This is also confirmed by the simulation experiments. The total GE channel model is

$$
P=\left[\begin{array}{cc}
\beta & 1-\beta \\
1-\alpha & \alpha
\end{array}\right]
$$

where $\alpha=1-(1-\beta)(1-p) / p$ and $\beta=\beta_{\text {out }} \beta_{\text {in }}$.

So we get the final PLR for all receivers, shown in formula (29).

$$
P L R(r)=P[m+1, m+1]=p \alpha^{m}
$$

Now we consider the expected number of transmissions for one multicast data packet for all receivers. The probability that $N>n$ is shown as follows.

$$
\begin{gathered}
P[N>0]=1 \\
P[N>1]=p_{\text {out }}+\left(1-p_{\text {out }}\right)\left(1-\left(1-p_{\text {in }}\right)^{R}\right) \\
P[N>n] \approx P_{\text {out }}[n, n]+\left(1-p_{\text {out }}\right)\left(1-\left(1-\left(p_{\text {in }} / p\right) P[n, n]\right)^{R}\right) \\
+\sum_{i=1}^{n-1}\left(P_{\text {out }}[n-i, n-i]\left(1-\alpha_{\text {out }}\right)\left(1-\left(1-\left(p_{\text {in }} / p\right) P[i, i]\right)^{R}\right)\right) \\
=p_{\text {out }}\left(\alpha_{\text {out }}\right)^{n-1}+\left(1-p_{\text {out }}\right)\left(1-\left(1-p_{\text {in }} \alpha^{n-1}\right)^{R}\right) \\
+\sum_{i=1}^{n-1}\left(p_{\text {out }}\left(\alpha_{\text {out }}\right)^{n-i-1}\left(1-\alpha_{\text {out }}\right)\left(1-\left(1-p_{\text {in }} \alpha^{i-1}\right)^{R}\right)\right)
\end{gathered}
$$


Finally the expected number of transmissions for one multicast data packet for all receivers can be calculated as in formula (33).

$$
\begin{gathered}
E[N]=\sum_{n=0}^{m} P[N>n] \\
\approx 1+\sum_{n=1}^{m}\left(\begin{array}{l}
p_{\text {out }}\left(\alpha_{\text {out }}\right)^{n-1}+\left(1-p_{\text {out }}\right)\left(1-\left(1-p_{\text {in }} \alpha^{n-1}\right)^{R}\right) \\
+\sum_{i=1}^{n-1}\left(p_{\text {out }}\left(\alpha_{\text {out }}\right)^{n-i-1}\left(1-\alpha_{\text {out }}\right)\left(1-\left(1-p_{\text {in }} \alpha^{i-1}\right)^{R}\right)\right)
\end{array}\right)
\end{gathered}
$$

Note that it is direct and simple to calculate the average channel holding time of BLBP from the expected number of transmissions in practice. We will evaluate the performance of BLBP and compare BLBP with LBP and different application layer multicast error control schemes by simulation experiments in the following section.

\section{Performance Evaluation}

In this section, we first evaluate the performance of BLBP and confirm the theoretical results by simulation experiments. Then we compare BLBP with the application layer multicast error control schemes HECPR and HARQ Type I. The metrics used for evaluation include the average number of transmissions, the maximum multicast delay and the total RI. Consistent with many references $[8,10,14]$, we also consider the redundant transmission only as RI.

Table 1. Application targets and parameters

\begin{tabular}{|c|c|}
\hline PLR Requirement & $1 \mathrm{e}-6$ \\
\hline Delay Constraint & $20-100 \mathrm{~ms}$ \\
\hline RTP Payload Length & $1316 \mathrm{Bytes}$ \\
\hline Multicast load interval & $2.5 \mathrm{~ms}$ \\
\hline RTT & $\approx 3.5 \mathrm{~ms}$ \\
\hline Original Error Rate & $\leq 10 \%$ \\
\hline Packet sent & $40-100 \mathrm{e} 6$ \\
\hline
\end{tabular}

Table 2. The retransmission limit and the temporal error correlation (PLR constraint 1e-6)

\begin{tabular}{|c|c|c|c|c|c|c|}
\hline \multirow{2}{*}{$\begin{array}{c}\text { Error } \\
\text { Rates }\end{array}$} & \multicolumn{7}{|c|}{ The temporal error correlations } \\
\cline { 2 - 7 } & 0.0 & 0.1 & 0.2 & 0.3 & 0.4 & 0.5 \\
\hline 0.05 & 4 & 6 & 8 & 10 & 13 & 17 \\
\hline 0.10 & 6 & 7 & 10 & 12 & 15 & 20 \\
\hline
\end{tabular}

We conduct our simulation study using ns-2 and implement BLBP based on the 802.11e simulation model from [17]. All client nodes are one hop to the AP and at most two hops to each other. We use IEEE 802.11a parameters to model the physical layer. The data rate we choose is $24 \mathrm{Mbps}$. The first receiver that joins the multicast group acts as the leader. The application targets and parameters are presented in Table 1. Note that the PLR target (1e-6) is very strict. We use this final PLR target as the MAC layer PLR target. The total payload length in the MAC layer is 1356 bytes, and there is no fragmentation in the MAC layer or the network layer. The retransmission limit of BLBP is determined following only the PLR constraint shown in formula (15). Some examples are shown in Table 2. We guarantee the PLR target first and explore the total multicast delays of BLBP in different network scenarios and channel conditions.

The application layer multicast error control schemes HEC-PR and HARQ Type I are implemented based on the real-time transport protocol (RTP) [18]. We use unicast for the feedback in HEC-PR and HARQ Type I instead of broadcast because unicast is more reliable. The simplified GE channel model is implemented in the physical layer, but it is used only for data frames. The MAC control frames (RTS/CTS/Beacon/ACK) are error free from the error model. (The control frames also may be lost because they might collide with the background traffic.) This also makes sense in practice because the control frames are very small and are sent in the lowest data rate, and hence they are more reliable than data frames.

First we compare the average numbers of transmissions for BLBP and LBP in different channel conditions. The results are shown in Figure 4. The simulation result and the theoretical result of BLBP match very well. As expected, BLBP is more efficient than LBP particularly when the error rates are high and the numbers of receivers are large. This is because BLBP allows all receivers to send feedback based on sequence check thanks to the beacon frame and LBP can not.

Then we explore the effect of the temporal error correlation. The average numbers of transmissions and the maximum multicast delays of BLBP with different temporal error correlations are shown in Figure 5 and Figure 6 respectively. About the expected number of transmissions, we can see that the theoretical analysis and the simulation results match very well. The temporal error correlation affects the average number of transmissions very much. However, the multicast delays are still very low even when the temporal error correlations are high, thanks to the fast ACK and retransmission in the MAC layer. So BLBP is very good for real-time multicast applications with strict delay constraints.

Figure 7 shows the average numbers of transmissions for BLBP with different spatial error correlations. The theoretical analysis and the simulation result match very well. BLBP can take full advantage of the spatial error correlation because of the complete feedback suppression thanks to the JACK scheme.

Then we compare BLBP with the application layer multicast error control schemes. The temporal error correlation and the spatial error correlation are set to 0.10 and 0.20 respectively to simulate a near realistic channel condition. First we compare BLBP with HECPR in different channel conditions. The total RIs and maximum multicast delays are shown in Figure 8 and 
Figure 9 respectively. When the error rates are high, we can see BLBP is much more efficient than HEC-PR which is a pure ARQ based scheme (without FEC coding). This is because BLBP suppresses the multiple feedbacks into just a JACK and it is more efficient than the application layer feedback and retransmission suppression in HEC-PR. The results also show that the multicast delays of BLBP are much shorter than the delays in HEC-PR. This is due to the fast MAC layer $\mathrm{ACK} /$ retransmission in the MAC layer. Moreover, because of the much longer delays among each transmission or retransmissions, HEC-PR isn't much affected by the temporal error correlation.

Figure 10 shows the total RIs of BLBP, HEC-PR and HARQ Type I under different delay constraints. As shown in Figure 9, HEC-PR takes a long time and hence only satisfies long delay constraints. Both BLBP and HARQ Type I can satisfy all the delay constraints from $20 \mathrm{~ms}$ to $100 \mathrm{~ms}$. We can see that BLBP is more efficient than HARQ Type I when the delay constraints are short and the numbers of receivers are small. This is because that HARQ Type I has to switch to pure FEC scheme (no ARQ) when the delay constraint are very short, hence it is not efficient.

Finally we compare the total RIs of BLBP, HEC-PR and HARQ Type I with the heterogeneity of receivers. The error rate for receiver 1 is variable and all the other receivers have a fixed error rate 0.01 . Figure 11 shows the result. We can see that both BLBP and HEC-PR are much more efficient than HARQ Type I because HARQ Type I has to set FEC code and other parameters according to the receiver with the worst channel condition but BLBP and HEC-PR are more adaptive. Moreover, due to the effect of the temporal error correlation (shown in Figure 5), here BLBP is a little less efficient than HEC-PR.

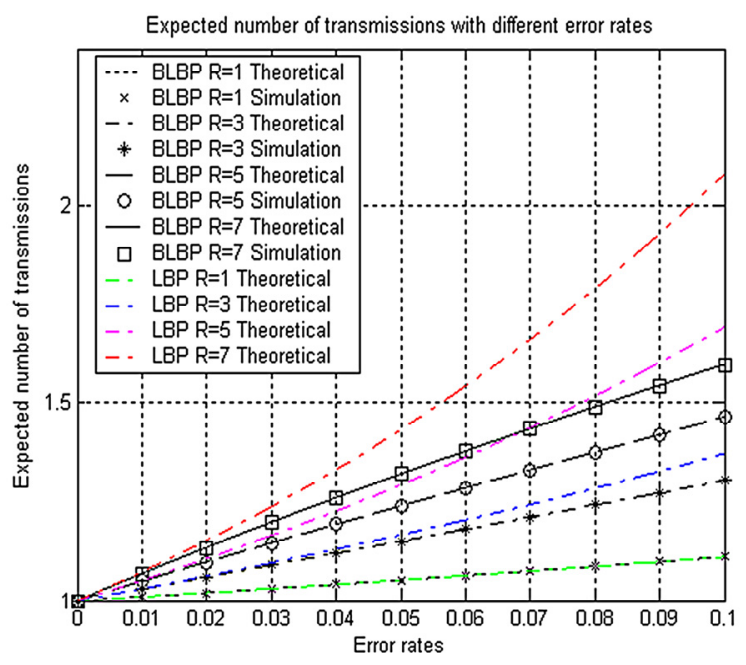

Figure 4. The expected number of transmissions with different error rates ( $\lambda=0 \quad \tau=0$ for all receivers)

\section{Conclusions}

In this work, we enhance LBP and propose BLBP for the MAC layer multicast error control in wireless networks. The use of the beacon frame is to lead the non-leader receivers to set timers and to announce the data frame sequence. On erroneous reception of a data frame that has not been correctly received before, the leader does not send an ACK, prompting a retransmission. On erroneous reception (The timer times out) of the data frame that has not been correctly received before, the non-leader receivers send NACKs to collide with the potential ACK from the leader, thus prompting the AP to retransmit the packet. To guarantee a very low PLR under strict delay constraints for video multicast over a GE channel with both the temporal error correlation and the spatial error correlation, we analyze BLBP and evaluate its performance via simulation experiments.

Both the theoretical analysis and simulation results show that BLBP can correct nearly all the errors for all receivers in the MAC layer. BLBP needs the minimum number of redundancy transmissions among all pure ARQ based schemes. BLBP is more efficient than the application layer ARQ schemes and the total multicast delay is much shorter. BLBP is even more efficient than the best application layer multicast error control scheme when the delay constraints are short or with the heterogeneity of receivers. BLBP is very good for the real-time multicast applications with strict delay constraints, especially for small groups.

In the future, we plan to extend BLBP with FEC coding and further improve the QoS of multicast cross the application layer and MAC layer in wireless networks.

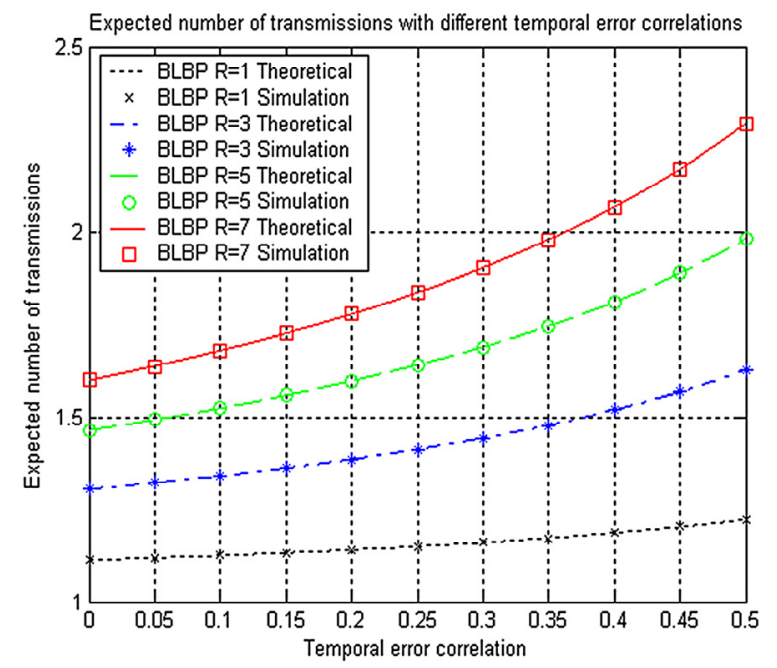

Figure 5. The expected number of transmissions with different temporal error correlations (error rate $0.10 \lambda=0$ for all receivers) 


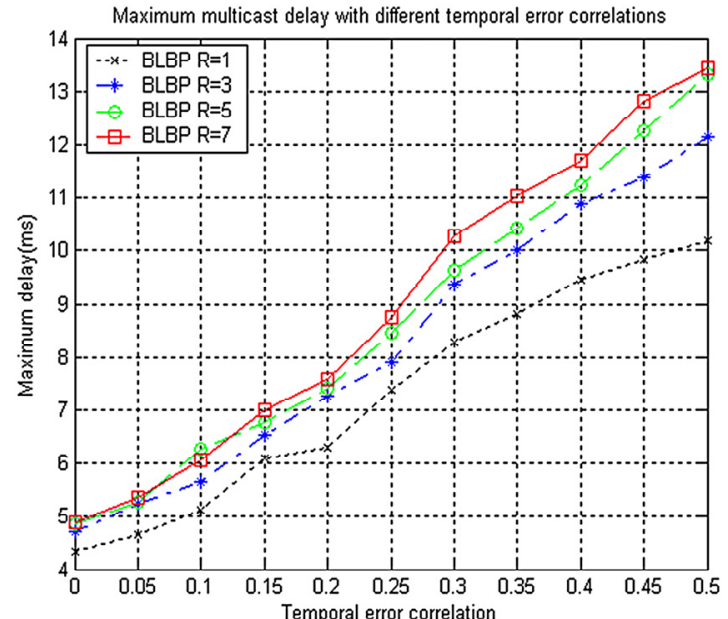

Figure 6. The maximum multicast delay with different temporal error correlations (error rate $0.10 \lambda=0$ for all receivers)

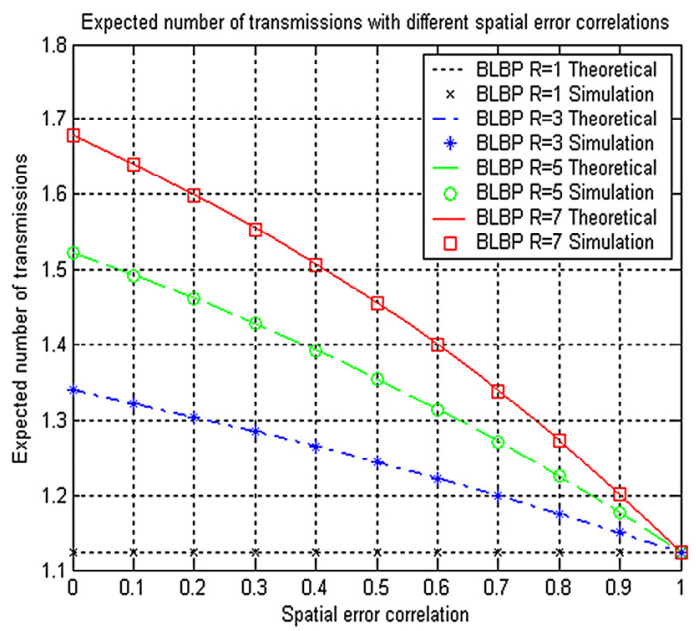

Figure 7. The expected number of transmissions with different spatial error correlations (error rate $0.10 \tau=\mathbf{0 . 1 0}$ for all receivers)

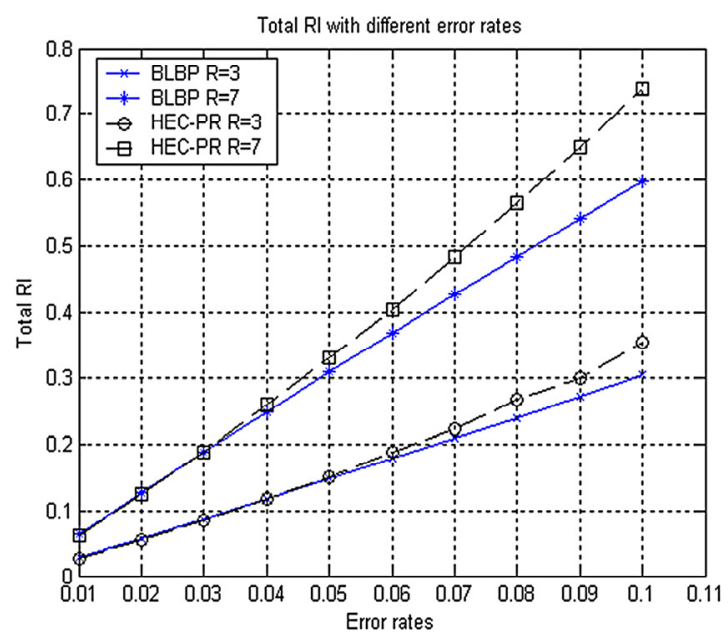

Figure 8. The total RI with different error rates $(\tau=\mathbf{0 . 1 0}$ $\lambda=0.20$ for all receivers)

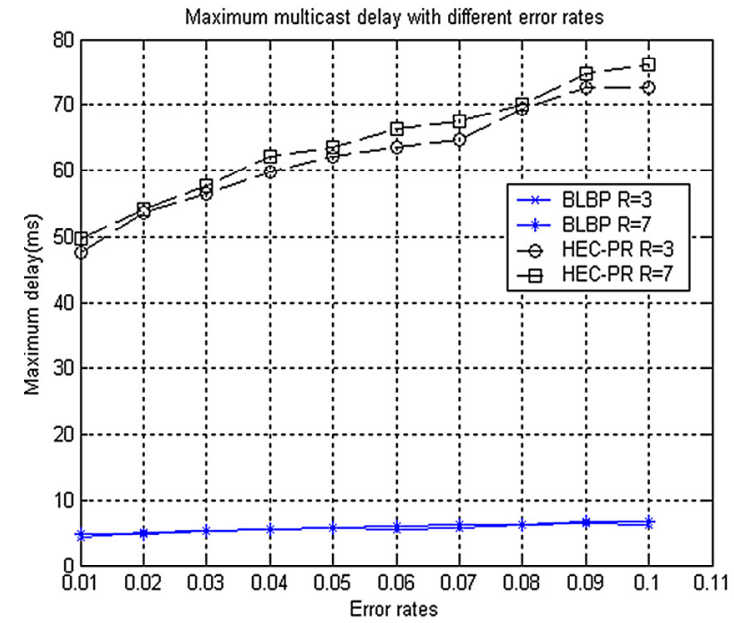

Figure 9. The maximum multicast delay with different error rates $(\tau=0.10 \quad \lambda=0.20$ for all receivers)

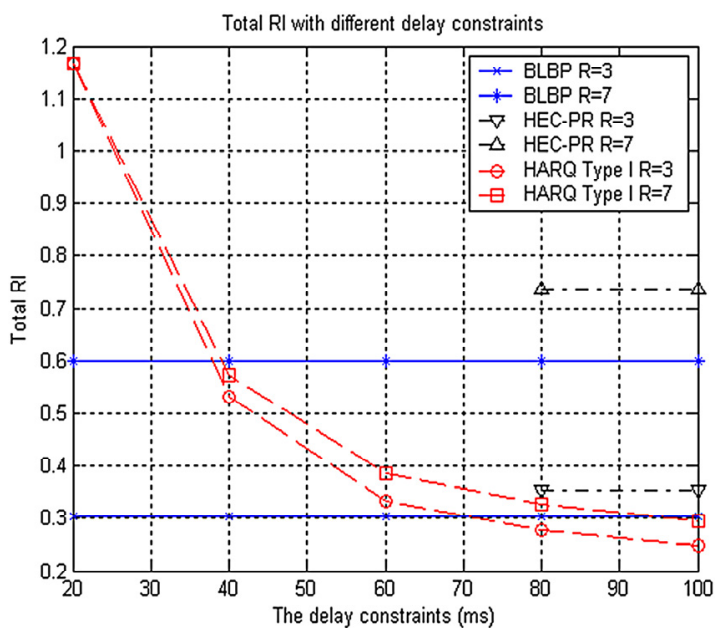

Figure 10. The total RI with different delay constraints (error rate $0.10 \tau=0.10 \lambda=0.20$ for all receivers)

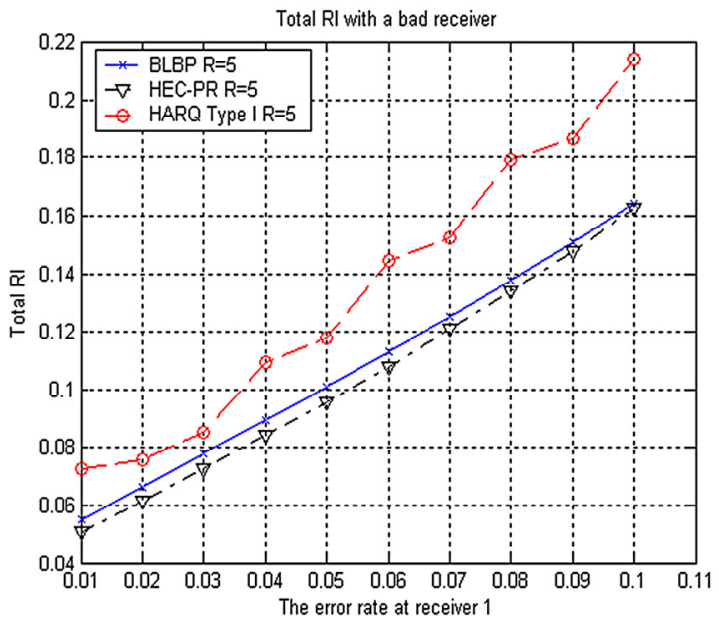

Figure 11. The total RI with a bad receiver 1 (error rate $0.01 \tau=0.10 \lambda=0.20$ for other receivers, delay constraint 100ms) 


\section{References}

[1] U.H. Reimers, "DVB-The Family of International standards for Digital Video Broadcasting," in Proceedings of IEEE, vol.94, no.1, pp. 173-182, January 2006.

[2] E.N. Gilbert, "Capacity of a burst-noise channel," Bell Syst. Tech. J., vol.39, pp. 1253-1265, September 1960.

[3] E.O. Elliott, "Estimates of error rate for codes on burstnoise channels," Bell Syst. Tech. J., vol.42, pp. 19771997, September 1963.

[4] M. Mushkin and I. Bar-David, "Capacity and coding for the Gilbert-Elliott channels," IEEE Trans. on Inform. Theory, vol. 35, pp. 1277-1290, November 1989.

[5] J. Nonnenmacher and E.W. Biersack, "Optimal multicast feedback," in IEEE Infocom, (San Francisco, California), pp. 964, March/April 1998.

[6] L. Rizzo, "Effective erasure codes for reliable computer communication protocols," ACM Computer Communication Review, April 1997.

[7] D. Qiao and K.G. Shin, "A two-step adaptive error recovery scheme for video transmission over wireless networks," in Proceedings of IEEE Infocom, 2000, March 2000.

[8] G.P. Tan and T. Herfet, "The Optimization of an RTP Level Hybrid Error Correction Scheme for DVB Systems in Wireless Home Networks under Strict Delay Constraint," IEEE Trans. on Broadcasting, November 2006.

[9] G. Carle and E.W. Biersack, "Survey of error recovery techniques for IP-based audio-visual multicast applications," IEEE Network, vol.11, no 6, pp. 24-36, November-December 1997.
[10] G. Tan and Th. Herfet, "Application Layer Hybrid Error Correction with Reed-Solomon Code for DVB Services over Wireless LANs," the 3rd IEEE International Conference on Wireless Communications, Networking and Mobile Computing (WiCOM), Shanghai, China, September 2007.

[11] J. Kuri, S.K. Kasera. "Reliable multicast in multi-access wireless LANs," in Proceedings of Infocom '99.

[12] J.Tourrilhes, "Robust broadcast: improving the reliability of broadcast transmission on CSMA/CA," in Proceedings of the Ninth IEEE International Symposium on. Personal, Indoor and Mobile Radio Communications, 1998.

[13] S.K.S. Gupta, V.Shankar, and S. Lalwani, "Reliable Multicast MAC Protocol for Wireless LANs," IEEE International Conference on Communications, vol. 1, pp. 93-97, 2003.

[14] J.R. Yee and E.J. Weldon, Jr., "Evaluation of the performance of error-correcting codes on a Gilbert channel", IEEE Tran. on Communications, vol. 43, no. 8, August 1995.

[15] G. Bianchi, "Performance analysis of the IEEE 802.11 distributed coordination function," IEEE Journal on Selected Areas in Communications, vol. 18, no. 3, March 2000.

[16] D.F. Towsley, J. Kurose, and S. Pingali, “A Comparison of Sender-Initiated and Receiver-Initiated Reliable Multicast Protocols," IEEE Journal on Selected Areas in Communications, vol. 15, no.3, April 1997.

[17] S. Wiethölter and C. Hoene, An IEEE 802.11e EDCA and CFB Simulation Model for ns-2, http://www.tkn.tuberlin.de/research/802.11e_ns2/.

[18] J. Ott, S. Wenger, N. Sato, C. Burmeister, and J. Rey, "Extended RTP profile for RTCP-based feedback," draftietf-avt-rtcp-feedback-11.txt, August 2004. 